\title{
Patterns and Predictors of Home Health and Hospice Use by Older Adults with Cancer
}

\author{
Julie L. Locher, $\mathrm{PhD}^{\star}, \dagger, \ddagger, \S, \|_{\star}$ Meredith L. Kilgore, $\mathrm{PhD}^{\dagger} \neq, \S$, Michael A. Morrisey, $\mathrm{PhD}^{\dagger}, \ddagger, \S$, \\ and Christine S. Ritchie, MD* ${ }^{*}, \boldsymbol{\uparrow}, \#$ \\ * Department of Medicine, Division of Gerontology and Geriatric Medicine, University of Alabama at \\ Birmingham, Birmingham, Alabama \\ † Center for Aging, University of Alabama at Birmingham, Birmingham, Alabama \\ ‡ Department of Health Care Organization and Policy, University of Alabama at Birmingham, \\ Birmingham, Alabama \\ $\S$ Lister Hill Center for Health Policy, University of Alabama at Birmingham, Birmingham, Alabama \\ I Department of Sociology, University of Alabama at Birmingham, Birmingham, Alabama \\ ף Center for Palliative Care, University of Alabama at Birmingham, Birmingham, Alabama \\ \# Birmingham/Atlanta Department of Veterans Affairs Geriatric Research, Education and Clinical \\ Center, Birmingham, Alabama
}

\begin{abstract}
OBJECTIVES-To describe patterns of home health and hospice use by older cancer patients and a comparison group of older persons without cancer. To identify predictors of home care and hospice utilization.
\end{abstract}

DESIGN—Retrospective analysis using the Surveillance, Epidemiology and End Results (SEER)Medicare Database, a linkage of the SEER Program of the National Cancer Institute (an epidemiological surveillance system of population-based tumor registries) and Medicare Claims.

SETTING-The SEER data used in this paper cover a service area that includes approximately $14 \%$ of the U.S. population, including the states of Connecticut, Hawaii, Iowa, and New Mexico and the metropolitan areas of Detroit, San Francisco-Oakland, Atlanta, Seattle-Puget Sound, Los Angeles County, and San Jose-Monterey.

PARTICIPANTS-Five analytical samples were drawn. The first consisted of all cases with a diagnosis of cancer in 1997 to 1999 who were eligible for services in calendar year 1999 ( $\mathrm{n}=$ 120,072). The second and third were subsamples of these and consisted of cases with a new cancer diagnosis in $1999(n=46,373)$ and cases who died in $1999(n=41,483)$. The fourth consisted of a comparison sample without cancer $(n=160,707)$. The fifth was a subsample of this and consisted of those who died in $1999(n=6,639)$.

MEASUREMENTS-Utilization rates of home health and hospice services.

RESULTS-Twenty-nine percent of cancer patients used home health services, and $10.7 \%$ used hospice services, compared with $7.8 \%$ of noncancer patients who used home health and less than $1 \%$

Address correspondence Julie L. Locher, PhD, 1530 3rd Avenue South, CH19-Room 219 N, Birmingham, AL 35294. jlocher@uab.edu. Author Contributions: All of the authors contributed to the study concept and design and preparation of the manuscript. The second author acquired the data, and the first and second authors analyzed and interpreted the data. 
who used hospice. Half (51.4\%) of cancer patients who used home health did not have cancer listed as an admitting diagnosis for the use of those services. Home health utilization was lowest for unmarried men.

CONCLUSION-This is the first study to evaluate community-based home health and hospice utilization by older cancer patients. Future studies must begin to address what constitutes appropriate utilization.

\section{Keywords}

home health; hospice; SEER-Medicare; database; cancer

Cancer disproportionately affects older adults, with age being the most significant risk factor for cancer. Currently, approximately 35 million people (13\% of the total population) in the United States are aged 65 and older. This number is expected to increase to nearly 80 million by $2050 .{ }^{1}$ The incidence rate for cancer is nearly 10 times greater in persons aged 65 and older than in those younger than 65 (2,208 vs 225 per 100,000 cases). ${ }^{2}$ Cancer is the second leading cause of death in persons aged 65 and older and is the primary cause of death in those aged 65 to $74 .{ }^{3}$ Despite declining or stabilizing cancer incidence rates across all age groups, the tremendous growth of the older population will contribute to a doubling of the current number of cancer cases by 2050, with the overwhelming majority of those cases occurring in older adults. ${ }^{4}$

Home health and hospice services can play a vital role in providing treatment for older cancer patients throughout the continuum of care of the disease, and both are covered under Medicare Part A. ${ }^{5}$ Home health services may be more appropriate during the earlier stages of illness, and hospice services may be more appropriate during the latter stages of illness. This is primarily because of the different emphases of the programs and the services that are offered. Home health services are provided for persons who are in need of care because of an acute illness, a chronic condition, a permanent disability, or a terminal illness. Care is limited to part-time or intermittent skilled nursing care, home health aide services, physical therapy, occupational therapy, speech-language services, medical social services, and some medical supplies and durable equipment. Hospice benefits are allowable only to persons with a terminal illness, and services provided include all of those offered under the home health benefit and many more, including coverage of drugs for symptom control and pain relief, grief counseling, respite care, and other services. Patients who are terminally ill may receive either type of service, but they may not receive home health and hospice services simultaneously.

Previous research has shown that a primary diagnosis of cancer accounts for $7.5 \%$ of all home health admissions for Medicare home health patients and that women and nonwhites are more likely to use home health services (not specific to cancer diagnosis) than men and whites, ${ }^{6,7}$ although this may significantly underestimate the proportion of older cancer patients receiving home health services, because the primary diagnosis recorded for a particular home health event may be related to sequelae or treatment of the cancer rather than the cancer itself. Additionally, many cancer patients have comorbid or chronic conditions, and these increase with age. ${ }^{8-10}$ The cancer or its treatment may exacerbate these conditions, and this may ultimately be the reason for referral to home health services. Adults with a prior history of cancer also have higher levels of disability than the general population of adults. ${ }^{11,12}$ Thus, a significant number of older adults with cancer receive home health services or could benefit from receipt of those services, although the precise numbers of older adults with cancer receiving these services is not known.

The majority (79.6\%) of patients who receive hospice services are aged 65 and older, and about half $(51.0 \%)$ of all Medicare hospice patients have a primary diagnosis of cancer. ${ }^{13}$ Previous 
estimates of hospice use by Medicare fee-for-service beneficiaries range from $19.8 \%$ to $30.2 \%$. 14,15 There is variation in the use of hospice services between patient subgroups; utilization has been found to be higher in patients who are married, female, and non-Hispanic white. ${ }^{15}$ It is not known whether persons who are terminally ill who do not receive hospice services also do not receive other services, such as home health services.

The Balanced Budget Act of 1997 (BBA) changed the reimbursement systems for home health and hospice services. After implementation of the BBA, which introduced a prospective payment system for home health services, use of home health services decreased and of hospice services increased until 2003 (the most recent year for which data are available from the Center for Medicare and Medicaid Services). ${ }^{6,16}$ The extent to which these utilization trends are related is not known. Although hospice utilization rates are known for older adults with cancer, their home health utilization rates are not known.

The primary aim of this article is to describe patterns of home health and hospice use by older cancer patients and a comparison group of older persons without cancer. Patterns of utilization are described for older cancer patients generally and for two phases of disease-immediately after diagnosis and immediately before death for patients who died. Patterns of utilization are described separately for a comparison group of older persons without cancer generally and immediately before death. Patterns of utilization are described in terms of total utilization and the proportion of utilization attributable directly to cancer. A secondary aim is to identify predictors of home care and hospice utilization, including cancer site and patient demographic characteristics of sex, marital status, and ethnicity.

\section{METHODS}

\section{Sample}

This paper uses the Surveillance, Epidemiology and End Results (SEER)-Medicare Database, a linkage of the SEER Program of the National Cancer Institute and the Medicare claims data for covered health services for individuals from the point of eligibility to death. ${ }^{17,18}$ The SEER program is an epidemiological surveillance system of population-based tumor registries designed to track cancer incidence and survival. Data collected include demographics (age, sex, and race), cancer-specific data (including date of diagnosis, type of cancer), follow-up vital status, and, if relevant, cause of death. The Medicare database includes claims data, including charges and reimbursements for Medicare-covered services (including home health and hospice services), and International Classification of Diseases, Ninth Revision (ICD-9), Healthcare Common Procedure Coding System, or Diagnostic Related Group codes to identify diagnoses and procedures. In addition, the SEER-Medicare database includes a 5\% random sample of Medicare beneficiaries residing in the SEER areas who do not have cancer. Demographic and Medicare claims data are collected for this sample and can be used for comparative purposes.

The SEER data used in this paper cover a service area that includes approximately $14 \%$ of the U.S. population, including the states of Connecticut, Hawaii, Iowa, and New Mexico and the metropolitan areas of Detroit, San Francisco-Oakland, Atlanta, Seattle-Puget Sound, Los Angeles County and San Jose-Monterey. The SEER data are highly valid, and the program's standard for completeness is $98 \% .{ }^{18}$ Medicare provides health insurance for approximately $96 \%$ of the population aged 65 and older, and $93.1 \%$ of older adults are covered by Medicare Parts A and B. Only persons covered by traditional Medicare Parts A and B and not enrolled in managed care (thus, only Medicare fee-for-service beneficiaries) are included in the analysis. The SEER-Medicare population is similar to the entire U.S. population aged 65 and older in terms of age and sex distribution but is less likely to be white (79.8\% vs $86.7 \%$ ), to be living in poverty (9.5\% vs $12.8 \%)$, or to experience cancer mortality ( 1,039 per 100,000 persons aged 
65 and older vs 1,128$)$ and more likely to be living in an urban area $(86.9 \%$ vs $73.0 \%)$ and participating in a health maintenance organization ( $26.0 \%$ vs $17.0 \%)$.

In each case, when individuals had been diagnosed with multiple cancers, the analysis uses the most recently diagnosed cancer site, because it is expected that the most recent cancer diagnosis would have the greatest influence on service utilization. In addition, because this article reports only on community-dwelling persons who would be eligible to receive home health or hospice services, persons who were admitted to a skilled or intermediate nursing facility for the long term were excluded from the samples. It is appropriate to use data from 1999, because by this time, the Interim Payment System authorized by the BBA had been fully implemented during fiscal year 1998, which began October 1, 1997, and ended September 30, 1998, and the effects of the eventual Prospective Payment System of the BBA in terms of utilization and costs of home health services were already apparent. ${ }^{19}$

Five analytical samples were drawn from the SEER-Medicare data for analysis. The first consisted of all cases who had their most recent cancer diagnosis in 1997, 1998, or 1999 and were eligible for services in calendar year $1999(n=120,072)$. The second and third were subsamples of these and consisted, respectively, of cases with a new cancer diagnosis in 1999 (with health service claims for 6 months after the date of diagnosis, $n=46,373$ ) and cases who died in 1999 (with claims for 6 months before the date of death, $n=41,483$ ). The fourth consisted of a random 5\% sample of Medicare beneficiaries residing in the SEER areas who did not have a diagnosis of cancer and who were eligible for services in calendar year 1999 (n $=160,707)$. The fifth was a subsample of the $5 \%$ of beneficiaries and consisted of those who died in $1999(n=6,639)$.

This research received approval from the University of Alabama at Birmingham institutional review board.

\section{Analysis}

Data analyses were performed using Stata v8 statistical software (Stata Corp., College Station, TX). Descriptive statistics were completed first describing the sample distributions for patient demographics and for the distributions of home health and hospice services by type of cancer to identify patterns of use. Estimates of the proportion of services attributable directly to cancer were also calculated. Service was defined as attributable to cancer if there was an ICD-9 code for malignancy. Next, expected utilization rates for each sample were estimated for patient subgroups (race, sex, and marital status) using probit regressions controlling for cancer type and stage to identify predictors of utilization.

\section{RESULTS}

Table 1 provides descriptive statistics for the full samples and the subsamples of newly diagnosed and deceased cases and the 5\% of noncancer cases along with the cases who died. The samples of cancer patients contained more men, married persons, and white patients. The most common cancer sites were prostate, breast, and colorectal in the full sample for the entire year. These sites, as well as lung cancer, were also common in patients who were newly diagnosed and had died. Of all persons with a diagnosis of cancer, $40.4 \%$ were identified as having early-stage disease (American Joint Committee on Cancer (AJC) Stage 0 or 1), and 9.3\% were identified as having late-stage disease (AJC Stage 4); thus, the majority of cases were in the intermediate stage.

Figures 1,2, and 3 illustrate the raw distribution of home health and hospice utilization between all patients with and without cancer and for specific cancer sites. For all persons without a diagnosis of cancer, $7.8 \%$ accessed home health services and fewer than $1 \%$ used hospice 
services. This contrasts with persons with a diagnosis of cancer, $29.0 \%$ of whom accessed home health services and $10.7 \%$ used hospice services. For the $29.0 \%$ of patients with a cancer diagnosis who used home health services, $51.4 \%$ of them did not have cancer listed as an admitting diagnosis for the use of those services. This pattern of utilization of home health services not directly attributable to cancer held across all cancer sites. Of subjects recently diagnosed with any cancer, $22.9 \%$ used home health and $4.1 \%$ used hospice services. Of those with cancer who died in 1999, 24.4\% used home health and 30.4\% used hospice services. This compares with $20.8 \%$ of persons who died in 1999 and did not have cancer who used home health and $12.7 \%$ who used hospice services.

The top 10 diagnoses for home health utilization among the 5\% sample without cancer included diabetes mellitus (11.6\%), pressure ulcers $(8.0 \%)$, heart failure(5.9\%), hypertension (3.8\%), stroke (3.8\%), osteoarthritis (3.6\%), chronic obstructive pulmonary disease (COPD) (2.7\%), dyspnea (2.3\%), pneumonia (2.0\%), and hip fracture (1.8\%), and the top five diagnosis for hospice utilization included heart failure (13.5\%), COPD (9.7\%), organic dementia $(9.4 \%)$, Alzheimer's disease (8.9\%), and ill-defined causes (7.3\%). This compares with the top 10 diagnoses for all cancer patients (excluding cancer as a diagnosis): diabetes mellitus (8.8\%), pressure ulcers $(5.7 \%)$, heart failure $(5.6 \%)$, stroke $(3.9 \%)$, osteoarthritis $(3.1 \%)$, COPD (3.1\%), hypertension (2.9\%), open wound (2.8\%), pneumonia (2.8\%), and hip fracture $(2.1 \%)$. In the periods after diagnosis and before death, the primary admitting diagnosis for home health and hospice was most likely to be attributable to cancer, but there was still a nonnegligible proportion of utilization not attributable to cancer.

For all persons included in the 5\% sample, the mean number of days spent in home health was 63.5 , the median was 36.0, and the interquartile range was 18.0 to 71.0 . The mean number of days spent in hospice was 46.0 , the median was 15 , and the interquartile range was 5.0 to 60.0 . The majority ( $80.5 \%$ ) of the $5 \%$ sample who used hospice services received only home hospice care; the remainder had inpatient and at-home care. For all patients with cancer, the mean number of days spent in home health was 50.0, the median was 31.0 , and the interquartile range was 15.0 to 60.0. The mean number of days spent in hospice was 38.0, the median was 18.0, and the interquartile range was 7.0 to 46.0 . Nine percent of cancer patients received only inpatient hospice care, $80 \%$ received only home hospice care, and the remainder had some combination of the two.

In each of the samples of cancer patients, utilization rates for home health and hospice services were highest for those diagnosed with pancreatic cancer and lowest for those diagnosed with prostate cancer. An exception to this occurred in the sample who were recently diagnosed, for whom utilization rates for home health services were lowest for those diagnosed with melanoma. For all persons with a diagnosis of cancer and for newly diagnosed cases, home health utilization rates were always higher than for hospice care. For terminal patients, hospice utilization rates were higher, with the exception of bladder cancer, prostate cancer, and lymphomas, for which the rates were nearly the same.

Figure 4 compares expected utilization rates by race, sex, and marital status for the full sample and with the subsamples of newly diagnosed and deceased individuals. Cancer site and stage, race, sex, and marital status (where appropriate) were controlled for in the analyses. Because of the sample size, all regression coefficients are statistically significant at $P<.001$, so $P$-values for individual differences are not reported. In the full sample, whites had lower expected rates of home health use and slightly higher expected rates of hospice utilization than nonwhites. Unmarried women had the highest expected rates of home health use and unmarried men had the highest expected rates of hospice use; whereas married men had the lowest rates of home health utilization and married women had the lowest rates of hospice utilization. 
For newly diagnosed cancers, hospice utilization is rare (about $4 \%$, as indicated in the raw data), ranging from $3.7 \%$ for married men to $4.9 \%$ for unmarried women (Figure 5). Nonwhites had higher expected utilization rates of home health care (26.4\%) than whites (22.3\%). Women had higher utilization than did men, and unmarried women and men had higher utilization rates than their married counterparts.

For all cancer patients who died in 1999, expected hospice utilization was higher than use of home care for every group but nonwhites, for whom hospice utilization and use of home care was nearly identical (Figure 5). Whites had higher hospice and lower home health utilization than nonwhites. For home health and hospice care, married women had the highest utilization rates, unmarried men had the lowest home health utilization rates, and unmarried men had the lowest hospice utilization rates.

\section{DISCUSSION}

These findings indicate that older adults with cancer were more than three times as likely to use home health services than a comparison group without cancer $(29.0 \%$ vs $7.8 \%)$ and that more than half $(51.4 \%)$ of that utilization was not attributable to cancer. Furthermore, the utilization that was not attributable to cancer in those with cancer was nearly double the total utilization for the comparison group without cancer (14.9\% vs $7.8 \%$ ). The finding that home health utilization is higher in older adults with cancer is not unexpected, although the amount of that utilization that is not attributable to cancer is not expected and cannot be explained by admitting diagnoses. With the exception of open sores as a diagnosis, the reasons for home health utilization between the two groups were nearly identical.

The additional utilization of home health services by those with cancer suggests that those with cancer may be experiencing greater morbidity than those without cancer. This morbidity may be associated with sequelae or treatment of the cancer. The additional finding that home health use is greater for all persons with a diagnosis of cancer compared than for those who have recently been diagnosed (and in need of services related to cancer treatment) or for those who have recently died (and in need of services related to end-of-life care) support the interpretation that excess morbidity may be related to sequelae or long-term effects of treatment of the cancer. These data were restricted to include only those with a cancer diagnosis in 1997, 1998, or 1999. Future researchers might examine the increased morbidity and health service utilization of cancer survivors - a group that has not been extensively studied — over a longer period of time. 20

In contrast to findings for home health utilization, hospice utilization rates of $30.4 \%$ for cancer patients in the last 6 months of life are consistent with those reported previously, although $24.4 \%$ of cancer patients in the current study used home health instead of hospice in the 6 months before death. Healthcare services ought to be provided based upon need and benefit from those services. These findings cannot determine whether hospice services may have been more appropriate for cancer patients who received home health services 6 months before death. Previous research has demonstrated that healthcare utilization and costs are most intensive at the end of life. Future research might investigate whether utilization of home health versus hospice services results in better outcomes (such as better symptom relief and attention to emotional, spiritual, and practical needs), less utilization of other services, or lower costs for all time periods.

Demographic characteristics predicted statistically significant differences in home health and hospice utilization, although the magnitude of these differences was rather small. An exception to this was the lower utilization rates of home health services by married men than by all other groups for all samples. Only community-dwelling patients were included in the analysis. It 
may be that, in the absence of a female caregiver at home, other groups rely upon other care arrangements-within the community (thus accounting for their higher utilization of home health services) or in long-term care institutions (something the findings cannot determine). Future research might investigate alternative care arrangements by different groups and the associated outcomes and costs of these arrangements. Included in these investigations ought to be a consideration of the caregiver burden of those providing care, especially spouses.

To the authors' knowledge, this is the first study to comprehensively evaluate communitybased home health and hospice utilization by older cancer patients. The real question that this study raises is: "What is the appropriate utilization of community-based services for older adults with cancer?" This is an important issue that others have raised in reference to transitions of care for all patients residing in the community as they transfer between different locations or different care providers and different levels of care. ${ }^{21,22}$ This matter is also consistent with the Institute of Medicine's recent report on cancer survivorship and their call for the need to improve understanding of health care of cancer survivors, including community-based care. ${ }^{22}$

Directions that future studies might take include addressing the optimal role of home health and hospice in cancer care in this population, including whether and when such services ought to be used, and how we can tell the difference. Future studies might also examine differences between geographical location and differences between fee-for-service and managed care in the utilization of home health and hospice services—-something that the present study was not able to determine.

\section{Acknowledgments}

The authors thank Steve Mennemeyer from the University of Alabama at Birmingham, Joan Warren from the National Cancer Institute, and two anonymous reviewers for their comments, which significantly improved the quality of the manuscript.

Financial Disclosure: This work was supported by an intramural grant from the University of Alabama at Birmingham Center for Aging and an extramural grant from the National Cancer Institute (R21 CA112668) to Meredith L. Kilgore. None of the authors have any financial conflict to report.

Sponsors' Role: The sponsors played no role in the design, methods, subject recruitment, data collection, analysis, or preparation of this manuscript.

\section{References}

1. Federal Interagency Forum on Aging-Related Statistics. Older Americans 2000: Key Indicators of Well-Being. Washington, DC: U.S. Government Printing Office; 2000.

2. U.S. Cancer Statistics, 1999 Incidence. National Cancer Institute Surveillance, Epidemiology, and End Results. [Accessed December 1, 2005]. Available at http://www.seer.cancer.gov

3. Anderson RN. Deaths: Leading causes for 2000. Natl Vital Stat Rep 2002;50:1-85. [PubMed: 12355905]

4. Edwards BK, Howe HL, Ries LAG, et al. Annual report to the nation on the status of cancer, 19731999, featuring implications of age and aging on U.S. cancer burden. Cancer 2002;94:2766-2792. [PubMed: 12173348]

5. Center for Medicare and Medicaid Services. Medicare and You. 2006 [Accessed December 1, 2005]. [on-line]Available at www.medicare.gov/publications/pubs/pdf/10050.pdf

6. Health Care Financing Administration, Office of Information Services. Health Care Financing Review: Medicare and Medicaid Statistical Supplement. 2003 [Accessed February 28, 2006]. [on-line] Available at www.cms.hhs.gov/apps/review/supp/2003/license_use.asp

7. Murtaugh CM, McCall N, Moore S, et al. Trends in Medicare Home Health Care Use: 1997-2001. Health Aff (Millwood) 2003;22:146-156. [PubMed: 14515890] 
8. Yancik R. Epidemiology of cancer in the elderly. Current status and projects for the future. Rays 1997;22:3-9. [PubMed: 9250005]

9. Yancik R, Ganz PA, Varricchio CG, et al. Perspectives on comorbidity and cancer in older patients: Approaches to expand the knowledge base. J Clin Oncol 2001;19:1147-1151. [PubMed: 11181680]

10. Yates JW. Comorbidity considerations in geriatric oncology research. CA Cancer J Clin 2001;51:329_ 336. [PubMed: 11760567]

11. Hewitt M, Rowland JL, Yancik R. Cancer survivors in the United States. Age, health, and disability. J Gerontol A Biol Sci Med Sci 2003;58A:M82-M91.

12. McNeil JM, Binette J. Prevalence of disabilities and associated health conditions among adultsUnited States, 1999. MMWR Morb Mortal Wkly Rep 2001;50:120-125. [PubMed: 11393491]

13. Hospice Facts and Statistics. National Association for Home Care and Hospice. Apr2004 [Accessed October 5, 2005]. [on-line]Available at www.nahc.org/NAHC/Research/04HPC_Stats.pdf

14. McCarthy EP, Burns RB, Ngo-Metzger Q, et al. Hospice use among Medicare managed care and feefor-service patients dying with cancer. JAMA 2003;289:2238-2245. [PubMed: 12734135]

15. Lackan NA, Ostir GV, Freeman JL, et al. Decreasing variation in the use of hospice among older adults with breast, colorectal, lung, and prostate cancer. Med Care 2004;42:116-122. [PubMed: 14734948]

16. National Center for Health Statistics. Data Warehouse on Trends in Health and Aging. [Accessed February 27, 2006]. [on-line]Available at www.cdc.gov/nchs/agingact.htm

17. SEER-Medicare Linked Database. [Accessed December 1, 2005]. [on-line]Available at http://healthservices.cancer.gov/seermedicare/

18. Warren JL, Klabunde CN, Schrag D, et al. Overview of the SEER-Medicare data: Content, research applications, and generalizability to the United States elderly population. Med Care 2002;40(Suppl 8):3-18.

19. McCall N, Petersons A, Moore S, et al. Utilization of home health services before and after the Balanced Budget Act of 1997. What were the initial effects? Health Serv Res 2003;38:85-106. [PubMed: 12650382]

20. Hewitt, M.; Greenfield, S.; Stoval, E., editors. From Cancer Patient to Cancer Survivor: Lost in Translation. Washington, DC: The National Academies Press; 2005.

21. Coleman EA, Berenson RA. Lost in translation. Challenges and opportunities for improving the quality of transitional care. Ann Intern Med 2004;141:533-536. [PubMed: 15466770]

22. Coleman EA, Smith JD, Frank JC, et al. Preparing patients and caregivers to participate in care delivered across settings: The Care Transitions Intervention. J Am Geriatr Soc 2004;52:1817-1825. [PubMed: 15507057] 

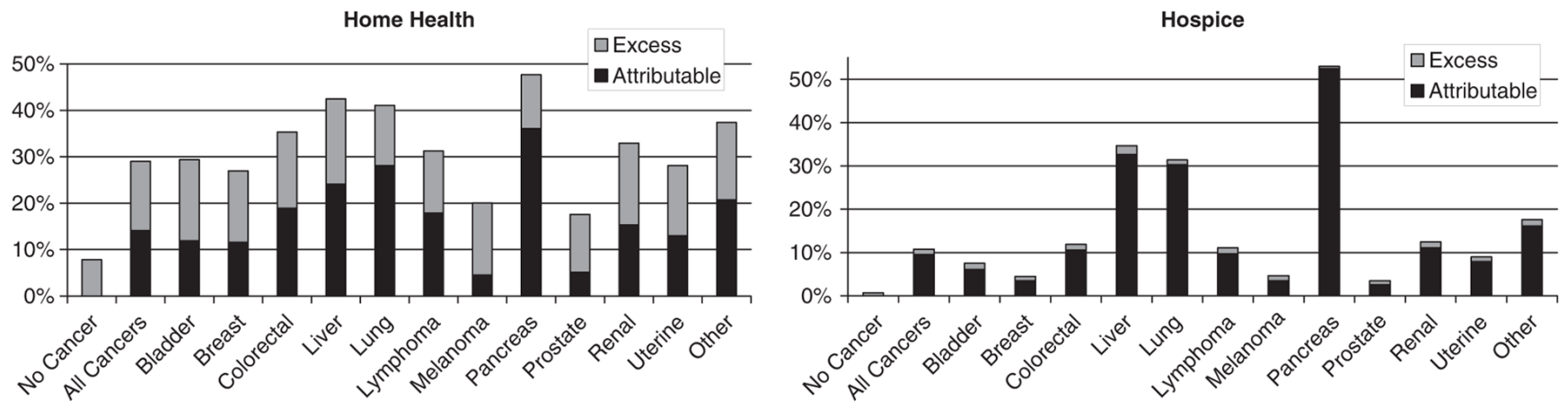

Figure 1.

Home health and hospice utilization by cancer site (full sample). 

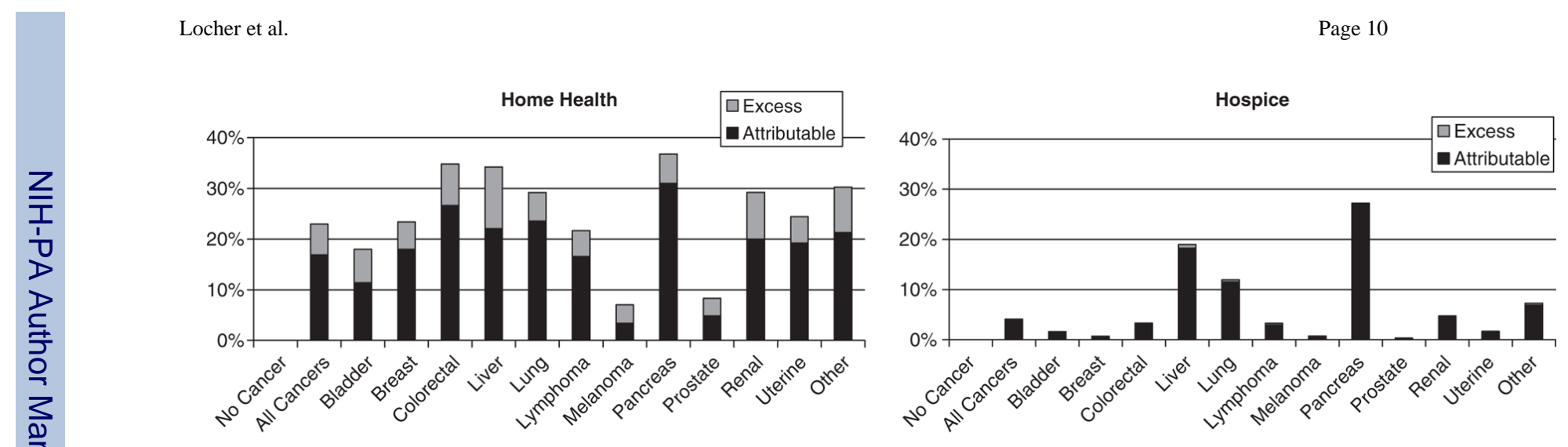

Figure 2.

Utilization within 6 months of cancer diagnosis. 

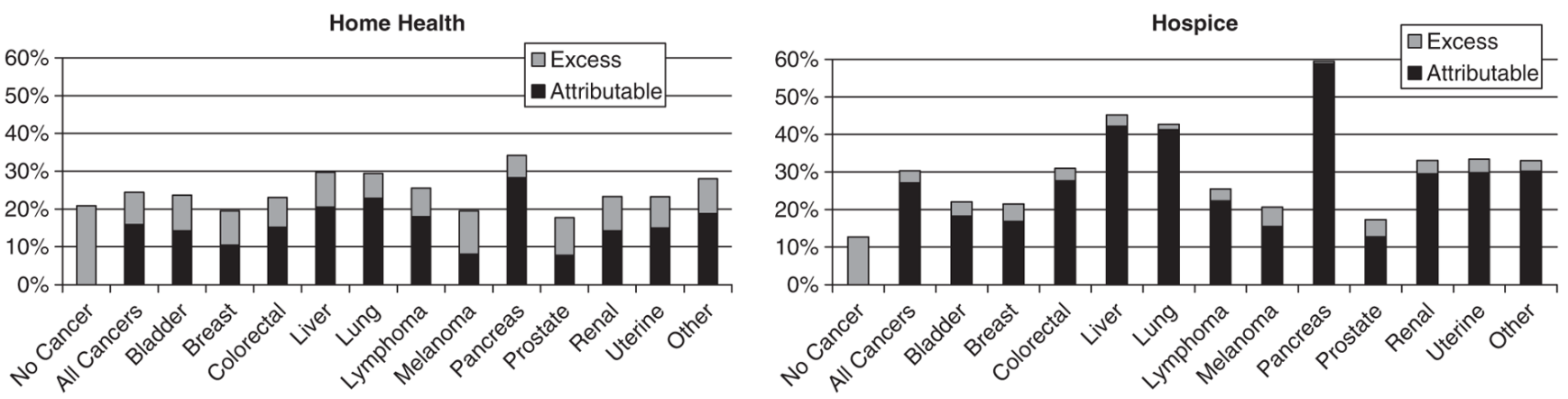

Figure 3.

Utilization within 6 months before death. 


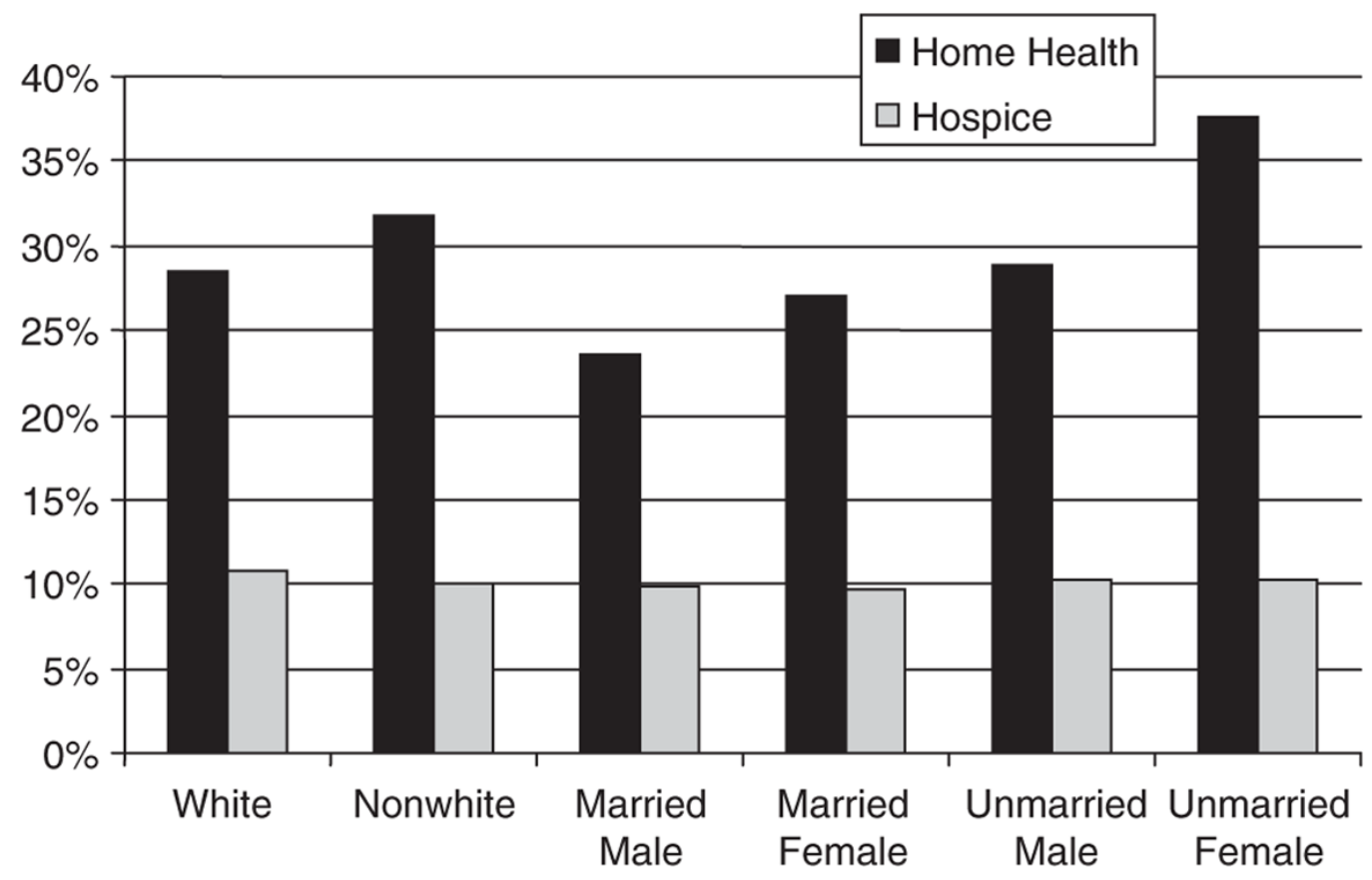

Figure 4.

Expected utilization among all cancer patients 1999. 


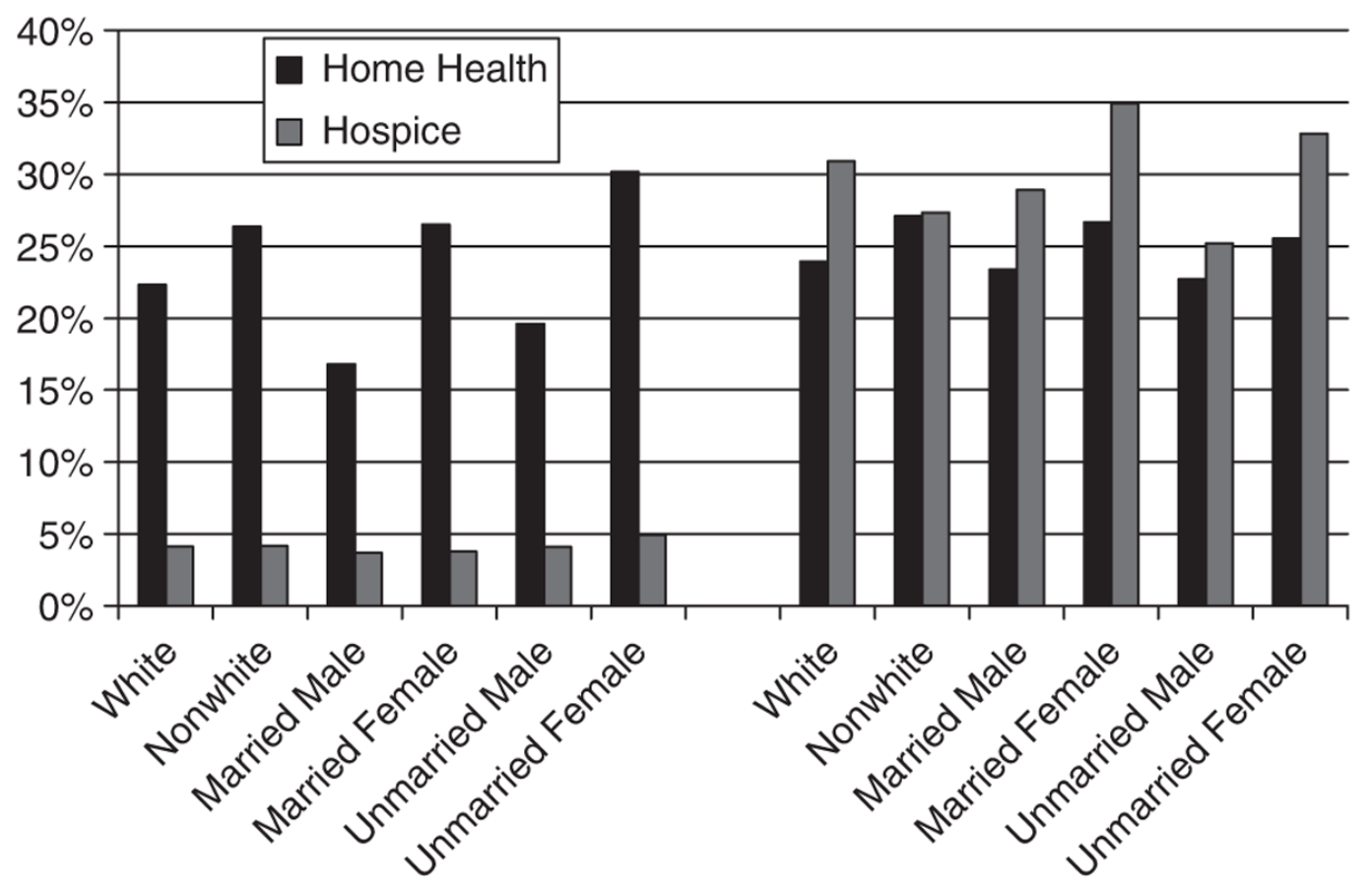

\title{
Six Months After
}

Diagnosis

\author{
Six Months Before Death
}

Figure 5.

Expected utilization for cancer patients. 


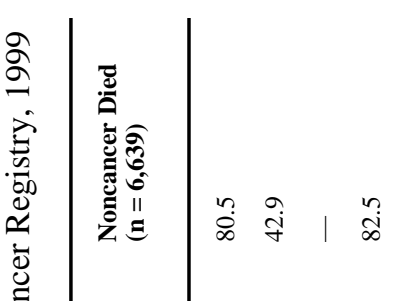

(a)

. 